\title{
Improved Hausdorff dimension estimate for vertical projections in the Heisenberg group
}

\author{
KATRIN FÄSSLER AND RISTO HOVILA
}

\begin{abstract}
We explore the effect of vertical projections on the Hausdorff dimension of sets in the Heisenberg group endowed with the Korányi distance. It is known that the dimension of an at most one-dimensional set generically does not decrease under such mappings. The proof uses a potential-theoretic approach which, for higher dimensional sets, only yields a trivial lower bound. In the present note, we provide an improved estimate for the dimension and thus prove that the previous trivial bound is not sharp. Moreover, for the larger family of projections onto cosets of vertical subgroups, we show that the potential-theoretic approach can be applied to establish almost sure dimension conservation for sets of dimension up to two.
\end{abstract}

Mathematics Subject Classification (2010): 28A78 (primary); 28A75, 53C17 (secondary).

The $n$-th Heisenberg group $\mathbb{H}^{n}$ is the space $\mathbb{C}^{n} \times \mathbb{R}$ endowed with the group law

$$
(z, t) *(\zeta, \tau)=(z+\zeta, t+\tau+2 \omega(z, \zeta))
$$

with $\omega(z, \zeta):=\sum x_{i}^{\prime} y_{i}-x_{i} y_{i}^{\prime}$ for $z=(x, y), \zeta=\left(x^{\prime}, y^{\prime}\right)$. Whenever convenient, we will identify $\mathbb{H}^{n}$ with $\mathbb{R}^{2 n+1}$ in the obvious way. A left-invariant metric on $\mathbb{H}^{n}$ can be defined by

$$
d_{H}: \mathbb{H}^{n} \times \mathbb{H}^{n} \rightarrow \mathbb{R}, \quad d_{H}(p, q):=\left\|q^{-1} * p\right\|_{H},
$$

with

$$
\|(z, t)\|_{H}:=\sqrt[4]{|z|^{4}+t^{2}} .
$$

We are concerned with the effect of certain projection-type mappings on the Hausdorff $\operatorname{dimension}_{\operatorname{dim}_{H}}$ of sets in the Heisenberg group, where $\operatorname{dim}_{H}$ is computed

K. F. was supported by the Academy of Finland, project number 252293. R. H. was supported by the Finnish Centre of Excellence in Analysis and Dynamics Research.

Received Settember 5, 2013; accepted in revised form May 23, 2014.

Published online February 2016. 
with respect to the Korányi metric $d_{H}$. The study of dimension estimates for such projections in the spirit of Marstrand's theorem for orthogonal projections $[10,12,13]$ has been initiated in [1] and generalized in [2] to higher dimensional Heisenberg groups, and it consists essentially of two parts: the study of horizontal and vertical projections. Whereas a rather complete description of the dimensional behaviour of horizontal projections has been obtained in $[1,2,9]$, important questions for vertical projections have remained open due to the more complicated form of these mappings; in particular, a sharp lower dimension bound has only been proved for sets up to dimension one. This bound yields a rough dimension estimate for sets of larger dimension. It has been conjectured that this trivial estimate is not optimal, but counterexamples proving the non-sharpness or even a general improvement have not been known. The aim of this note is to extend the existing bound in two ways, namely we prove that (i) if, instead of the usual vertical projections, the larger family of 'projections' onto cosets of vertical subgroups is considered, then almost surely dimension does not drop for sets with dimension in the range between one and two, (ii) for the usual family of vertical projections in the first Heisenberg group and sets with dimension between two and three, the trivial lower bound is not sharp and it can be improved by an $\varepsilon$.

For part (i), we follow the standard potential-theoretic approach and exploit the additional parameter to obtain finiteness of certain energy integrals even for exponents larger than one. The proof of part (ii) uses an approach which has been developed by $\mathrm{T}$. Orponen in [17] to establish Hausdorff dimension estimates for special families of orthogonal projections onto lines and planes in $\mathbb{R}^{3}$.

In this paper, we consider the vertical projections $P_{\mathbb{V}^{\perp}}: \mathbb{H}^{n} \rightarrow \mathbb{V}^{\perp}$, for $V \in$ $G_{h}(n, m)$, which arise from the semi-direct group splitting

$$
\mathbb{H}^{n}=\mathbb{V}^{\perp} * \mathbb{V}
$$

with

$$
\mathbb{V}^{\perp}=V^{\perp} \times \mathbb{R} \text { and } \mathbb{V}=V \times\{0\} .
$$

Here, $G_{h}(n, m)$ denotes the Grassmannian of $m$-dimensional isotropic subspaces of $\mathbb{R}^{2 n}$. In the case $n=1$, we also write $\mathbb{V}_{\theta}^{\perp}=V_{\theta}^{\perp} \times \mathbb{R}$ and $\mathbb{V}_{\theta}=V_{\theta} \times\{0\}$ with $V_{\theta}=\operatorname{span}\left\{\left(\begin{array}{c}\cos \theta \\ \sin \theta\end{array}\right)\right\}$. Where convenient, we identify $\mathbb{V}$ with $V$. The pairs $(\mathbb{V}, *)$ and $\left(\mathbb{V}^{\perp}, *\right)$, respectively, are subgroups which are closed under the intrinsic Heisenberg dilations $\delta_{r}(z, t)=\left(r z, r^{2} t\right), r>0$. The formula $(0.1)$ for the group law yields

$$
P_{\mathbb{V}^{\perp}}(z, t)=\left(\pi_{V^{\perp}}(z), t-2 \omega\left(\pi_{V^{\perp}}(z), \pi_{V}(z)\right)\right),
$$

where $\pi_{V}$ denotes the orthogonal projection from $\mathbb{R}^{2 n}$ onto the subspace $V \in$ $G_{h}(n, m) \subseteq G(2 n, m)$, and analogously, $\pi_{V^{\perp}}$ is the projection on the Euclidean orthogonal complement of $V$ in $\mathbb{R}^{2 n}$. Each point $p=(z, t)$ in the Heisenberg group can be written in a unique way as a product $p=p_{\mathbb{V} \perp} * p_{\mathbb{V}}$ with $p_{\mathbb{V} \perp}=P_{\mathbb{V} \perp}(p)$ and $p_{\mathbb{V}}=P_{\mathbb{V}}(p):=\left(\pi_{V}(z), 0\right)$. We are concerned with projections not only onto vertical subgroups $\mathbb{V}^{\perp}, V \in G_{h}(n, m)$, but also on cosets of such (normal) subgroups, that is, on $u * \mathbb{V}^{\perp}=\mathbb{V}^{\perp} * u$ with $u \in \mathbb{V}=V \times\{0\}$. 
Euclidean orthogonal projections have the property that the result of first translating a set and then projecting is the same as first projecting and then translating by the projected translation vector. Given $u \in V$, it follows that each point $p$ can be written as a unique element

$$
p=\left(u+\pi_{V^{\perp}}(p)\right)+\pi_{V}(p-u) \in\left(u+V^{\perp}\right)+V
$$

and the projection $\pi_{V^{\perp}, u}$, which is defined by $\pi_{V^{\perp}, u}(p)=u+\pi_{V^{\perp}}(p)$ and which maps to the affine subspace $u+V^{\perp}$, has the property

$$
\operatorname{dim}_{E} \pi_{V^{\perp}, u}(B)=\operatorname{dim}_{E} \pi_{V^{\perp}}(B) .
$$

There is no corresponding statement for vertical projections on the Heisenberg group since these mappings are not group homomorphisms and right translations are not isometries with respect to $d_{H}$. For a fixed $V \in G_{h}(n, m)$ and $u \in V$, we write

$$
p=u * u^{-1} * p=u *\left(u^{-1} * p\right)_{\mathbb{V}^{\perp}} *\left(u^{-1} * p\right)_{\mathbb{V}}
$$

and set $p_{\mathbb{V}^{\perp}, u}=u *\left(u^{-1} * p\right)_{\mathbb{V} \perp}$ and $p_{\mathbb{V}, u}=\left(u^{-1} * p\right)_{\mathbb{V}}$. Then $p=p_{\mathbb{V}^{\perp}, u} * p_{\mathbb{V}, u}$ is the unique way of writing the point $p$ as an element in $\left(u * \mathbb{V}^{\perp}\right) * \mathbb{V}$ and we define the translated (or randomized) vertical projection $P_{\mathbb{V}^{\perp}, u}: \mathbb{H}^{n} \rightarrow u * \mathbb{V}^{\perp}=\mathbb{V}^{\perp} * u$ as

$$
P_{\mathbb{V}^{\perp}, u}(p)=u *\left(u^{-1} * p\right)_{\mathbb{V}^{\perp}}=p_{\mathbb{V}^{\perp}} * u=R_{u} \circ P_{\mathbb{V}^{\perp}}(p),
$$

where $R_{u}$ denotes right translation. In this way, we obtain a family with a larger parameter space. Even if it is not clear whether almost sure dimension conservation for sets of large dimension holds within the family of usual vertical projections, we can prove this property for 'generic' vertical projections $P_{\mathbb{V} \perp, u}$. The idea of proving that a property - which may fail in the deterministic case - holds 'generically' for 'randomized' elements in an appropriate family is not new; it appears for instance in context of dimension estimates for self-affine sets.

Returning to vertical projections in the Heisenberg group, the following lower dimension bound has been proved in [2].

Theorem 0.1 ([2]). Let $B \subset \mathbb{H}^{n}$ be a Borel set with $\operatorname{dim}_{H} B \leq 1$. Then

$$
\operatorname{dim}_{H} P_{\mathbb{V}^{\perp}}(B) \geq \operatorname{dim}_{H} B \quad \text { for } \mu_{n, m} \text { almost every } V \in G_{h}(n, m),
$$

and this bound is sharp.

The proof uses the potential-theoretic method that has been developed by $\mathrm{R}$. Kaufman for planar orthogonal projections. In the context of vertical projections on the Heisenberg group, this technique can only be applied for sets up to dimension one, as certain integral averages of energies might blow up otherwise. Hence, a priori, we only have the trivial almost sure bound $\operatorname{dim}_{H} P_{\mathbb{V} \perp}(B) \geq 1$ for sets $B C$ $\mathbb{H}^{n}$ with $\operatorname{dim}_{H} B>1$.

Our first result improves this bound for randomly translated projections. 
Theorem 0.2. For every Borel set $B \subset \mathbb{H}^{n}$ with $\operatorname{dim}_{H} B \leq 2$, one has for $\mu_{n, m}$ almost every $V \in G_{h}(n, m)$,

$$
\operatorname{dim}_{\mathrm{H}} B_{V, u} \geq \operatorname{dim}_{\mathrm{H}} B \quad \text { for } \mathcal{H}^{m} \text { almost every } u \in \mathbb{V},
$$

where $B_{V, u}:=P_{\mathbb{V}^{\perp}, u}(B)$.

Our second result shows that the lower bound for the dimension obtained in [1] for vertical projections in the first Heisenberg group is not sharp. The existing bound from [1] reads

$$
\operatorname{dim}_{H} B_{\theta} \geq\left\{\begin{array}{lr}
\operatorname{dim}_{H} B & \text { if } 0 \leq \operatorname{dim}_{H} B \leq 1 \\
1 & \text { if } 1 \leq \operatorname{dim}_{H} B \leq 3 \\
2 \operatorname{dim}_{H} B-5 & \text { if } 3 \leq \operatorname{dim}_{H} B \leq 4
\end{array} \quad \text { for almost every } \theta\right.
$$

where the case $0 \leq \operatorname{dim}_{H} B \leq 1$ is included in Theorem 0.1 . We improve this bound by an $\varepsilon$ for sets with $2<\operatorname{dim} B \leq 3.00348$.

Theorem 0.3. Let $2<s$. Then there exists $\alpha(s)>0$ such that for every Borel set $B \subset \mathbb{H}^{1}$ with $\operatorname{dim}_{\mathrm{H}} B=s$, one has

$$
\operatorname{dim}_{\mathrm{H}} B_{\theta} \geq 1+\alpha(s) \text { for almost every } \theta \in[0, \pi),
$$

where $B_{\theta}:=P_{\mathbb{V}_{\theta}^{\perp}}(B)$.

Remark 0.4. The proof shows that we may take

$$
\alpha(s)=\frac{(s-2)(s-1)}{32 s^{2}}
$$

but this is conjecturally not sharp. With this choice for $\alpha(s)$, the bound from Theorem 0.3 is better than the one from (0.1) for $2 \leq s \leq 3.00348$.

We conclude this introduction by remarking that homogeneous subgroups and the related projections play an important role in the geometric measure theory on the Heisenberg group as counterparts for vector subspaces and orthogonal projections in Euclidean spaces, as can be seen from recent research articles in the area. To name but a few, we mention $[4,8,11,15]$.

Acknowledgements. We are grateful to Pertti Mattila and Tuomas Orponen for helpful comments and their interest in this project. We would like to thank them and David Preiss for the discussions which led to the present article. We also thank the referee for valuable remarks. 


\section{Preliminaries}

A word concerning notation first. We will write $a \lesssim b$, if $a \leq C b$ for some constant $C \geq 1$. If necessary, we will specify on which parameters $C$ is allowed to depend. Typically, it may depend on data which has been fixed at the beginning of the proof, but not for instance on the scale $\delta$ in a multi-scale argument. By $B(p, r)$ we denote a closed ball with respect to the Heisenberg metric. The closed $\delta$-neighbourhood of a set $A$ with respect to $d_{H}$ is written as $N_{H}(A, \delta)$, whereas $N_{E}(A, \delta)$ denotes the closed metric neighbourhood of the same set with respect to the Euclidean distance. The Korányi distance and the Euclidean metric on the Heisenberg group and on the underlying Euclidean space, respectively, are not bi-Lipschitz equivalent; however, we have the following local result (see [3, Lemma 2.1]).

Lemma 1.1. Let $A \subset \mathbb{R}^{3}$ be a set contained in the Euclidean ball of radius $r$ centred at the origin. Then there exist constants $0<c_{1}=c_{1}(r)<c_{2}=c_{2}(r)<\infty$ such that for all $p, q \in A$, we have

$$
c_{1} d_{E}(p, q) \leq d_{H}(p, q) \leq c_{2} d_{E}(p, q)^{1 / 2} .
$$

The symbols $\mathcal{H}_{H, \delta}^{s}$ and $\mathcal{H}_{H}^{s}$ stand for the $s$-dimensional Hausdorff premeasure and measure with respect to $d_{H}$, respectively. Restricted to a horizontal subgroup, the metrics $d_{E}$ and $d_{H}$ coincide, and in this case we may omit the subscript $H$ for the Hausdorff measures. We write $\mathcal{M}(A)$ for the collection of positive, finite Borel regular measures supported on a set $A$. The Hausdorff dimension of $A$ is related to the existence of a measure $\mu \in \mathcal{M}(A)$ satisfying a certain growth condition. This connection is the content of Frostman's lemma, which we recall in the following for the Heisenberg group. The general version for complete, separable metric spaces is due to Howroyd, see also [14].

Theorem 1.2 (Frostman's lemma). Let $B$ be a Borel subset of $\left(\mathbb{H}^{n}, d_{H}\right)$. Suppose that there exists $s>0, \mu \in \mathcal{M}(B)$, and $r_{0} \in(0, \infty]$ so that the inequality

$$
\mu(B(p, r)) \leq r^{s}
$$

holds for all $p \in B$ and $0<r<r_{0}$. Then $\mathcal{H}_{H}^{s}(B)>0$. In particular, $\operatorname{dim}_{H} B \geq s$. Conversely, if $\mathcal{H}_{H}^{s}(B)>0$ then there exists $\mu \in \mathcal{M}(B)$ so that (1.2) holds for all $p \in B$ and $r>0$.

Theorem 1.3 (Frostman's lemma: energy version). Let $B$ be a Borel subset of $\left(\mathbb{H}^{n}, d_{H}\right)$ and let $s>0$ be such that there exists a mass distribution $\mu \in M(A)$ with finite $s$-energy

$$
I_{s}(\mu):=\iint d_{H}(p, q)^{-s} \mathrm{~d} \mu(p) \mathrm{d} \mu(q)<\infty .
$$

Then $\operatorname{dim}_{H} B \geq s$. Conversely, if $B$ is a Borel subset of $\left(\mathbb{H}^{1}, d_{H}\right)$ and $s<\operatorname{dim}_{H} B$, then there exists $\mu \in M(A)$ with $I_{s}(\mu)<\infty$. 


\section{Proof of Theorem 0.2}

Proof of Theorem 0.2 . If the set $B$ is written as a countable union of subsets, countable stability of the Hausdorff dimension allows us to verify the dimension bound for each of the sets in the union individually. Since for all $V \in G_{h}(n, m)$ and all $u \in \mathbb{V}$, the projection $P_{\mathbb{V} \perp, u}$ restricted to the $t$-axis is an isometry, it is is sufficient to consider the part of $B$ which lies outside the $t$-axis. Using the countable stability of Hausdorff dimension, we can then assume that the set $B$ is bounded and consisting of points $p=(z, t)$ with $|z|$ bounded away from 0 and $\infty$. Further decomposing the domain if necessary, we may suppose that for all points $p=(z, t)$ and $q=(\zeta, \tau)$ in $B$, we have $|z+\zeta| \simeq 1$

The proof follows the standard potential-theoretic approach, namely, we start by choosing a Frostman measure $\mu \in \mathcal{M}(B)$ so that $I_{s}(\mu)<\infty$. Here, $s<$ $\operatorname{dim}_{H} B \leq 2$ and we will eventually let $s$ tend to $\operatorname{dim}_{H} B$ in order to complete the proof. Now, for fixed $V \in G_{h}(n, m)$ and $u \in V$, the measure $\mu_{V, u}$ which is defined by

$$
\mu_{V, u}(E):=\mu\left(P_{\mathbb{V}^{\perp}, u}^{-1}(E)\right), \quad E \subseteq u * \mathbb{V}^{\perp}
$$

belongs to $\mathcal{M}\left(B_{V, u}\right)$. Hence, if we can show that $I_{s}\left(\mu_{V, u}\right)<\infty$ almost surely, this will provide the generic lower bound $\operatorname{dim}_{H} B_{V, u} \geq s$.

To achieve this goal, we consider

$$
\begin{aligned}
& \int_{G_{h}(n, m)} \int_{V} I_{S}\left(\mu_{V, u}\right) \mathrm{d} \mathcal{H}^{m}(u) \mathrm{d} \mu_{n, m}(V) \\
& =\int_{G_{h}(n, m)} \int_{V} \int_{B_{V, u}} \int_{B_{V, u}} d_{H}(v, w)^{-s} \mathrm{~d} \mu_{V, u}(v) \mathrm{d} \mu_{V, u}(w) \mathrm{d} \mathcal{H}^{m}(u) \mathrm{d} \mu_{n, m}(V) \\
& =\int_{B} \int_{B}\left(\int_{G_{h}(n, m)} \int_{V} d_{H}\left(P_{\mathbb{V}^{\perp}, u}(p), P_{\mathbb{V}^{\perp}, u}(q)\right)^{-s} \mathrm{~d} \mathcal{H}^{m}(u) \mathrm{d} \mu_{n, m}(V)\right) \mathrm{d} \mu(p) \mathrm{d} \mu(q)
\end{aligned}
$$

and aim at bounding the inner double integral from above by a constant multiple of $d_{H}(p, q)^{-s}$ so that the whole expression becomes bounded by the finite number $I_{s}(\mu)$. Given points $p^{\prime}=\left(z^{\prime}, t^{\prime}\right)$ and $q^{\prime}=\left(\zeta^{\prime}, \tau^{\prime}\right)$ in $\mathbb{V}^{\perp}$ and $u \in \mathbb{V}$, it is easy to see that

$$
d_{H}\left(R_{u}\left(p^{\prime}\right), R_{u}\left(q^{\prime}\right)\right)=\sqrt[4]{\left|z^{\prime}-\zeta^{\prime}\right|^{4}+\left(t^{\prime}-\tau^{\prime}+4 \omega\left(z^{\prime}-\zeta^{\prime}, u\right)\right)^{2}}
$$

Hence, for $p=(z, t)$ and $q=(\zeta, \tau)$, we obtain

$$
\begin{aligned}
& d_{H}\left(P_{\mathbb{V}^{\perp}, u}(p), P_{\mathbb{V}^{\perp}, u}(q)\right) \\
& =d_{H}\left(R_{u}\left(P_{\mathbb{V} \perp}(p)\right), R_{u}\left(P_{\mathbb{V}^{\perp}}(q)\right)\right) \\
& \geq\left|t-\tau-2 \omega\left(\pi_{V^{\perp}}(z), \pi_{V}(z)\right)+2 \omega\left(\pi_{V^{\perp}}(\zeta), \pi_{V}(\zeta)\right)+4 \omega\left(\pi_{V^{\perp}}(z-\zeta), u\right)\right|^{\frac{1}{2}} \\
& =\left|t-\tau-2 \omega(z, \zeta)-2 \omega\left(z+\zeta, \pi_{V}(z-\zeta)\right)+4 \omega\left(\pi_{V^{\perp}}(z-\zeta), u\right)\right|^{\frac{1}{2}}
\end{aligned}
$$


By assumption, $z \neq-\zeta$. If $z=\zeta$, then

$$
d_{H}\left(P_{\mathbb{V} \perp, u}(p), P_{\mathbb{V}^{\perp}, u}(q)\right)=|t-\tau|^{\frac{1}{2}}=d_{H}(p, q)
$$

and the desired estimate holds even pointwise and for arbitrary $s>0$. From now on, we will therefore assume that $z \neq \pm \zeta$ and hence $\pi_{V^{\perp}}(z-\zeta)=0$ only for a $\mu_{n, m}$ zero measure set of parameters $V \in G_{h}(n, m)$. As we are integrating over $G_{h}(n, m)$, we may therefore safely suppose that $\pi_{V^{\perp}}(z-\zeta) \neq 0$.

A further assumption which we wish to make now is that $u$ takes values in $V \cap B(0, R)$ for some fixed $R>1$. Our conclusion will then hold for 'almost every $V \in G_{h}(n, m)$ and almost every $u \in V \cap B(0, R)$ ' which gives the desired result since $V=\bigcup_{n \in \mathbb{N}} V \cap B(0, n)$.

Using the assumption $|z+\zeta| \simeq 1$, we find

$$
\begin{aligned}
I & :=I(p, q):=\int_{G_{h}(n, m)} \int_{V \cap B(0, R)} d_{H}\left(P_{\mathbb{V}^{\perp}, u}(p), P_{\mathbb{V}^{\perp}, u}(q)\right)^{-s} \mathrm{~d} \mathcal{H}^{m}(u) \mathrm{d} \mu_{n, m}(V) \\
& \lesssim|z-\zeta|^{-\frac{s}{2}} \int_{G_{h}(n, m)} \int_{V \cap B(0, R)}\left|f(V)+4 \omega\left(\pi_{V^{\perp}}(v), u\right)\right|^{-\frac{s}{2}} \mathrm{~d} \mathcal{H}^{m}(u) \mathrm{d} \mu_{n, m}(V)
\end{aligned}
$$

with

$$
f(V):=\frac{t-\tau-2 \omega(z, \zeta)-2 \omega\left(z+\zeta, \pi_{V}(z-\zeta)\right)}{|z-\zeta||z+\zeta|},
$$

and

$$
v:=\frac{z-\zeta}{|z-\zeta|} .
$$

It is possible that $v$ is such that $\omega\left(\pi_{V^{\perp}}(v), u\right)=0$ for all $u \in V$, namely if $v$ lies in the intersection of the symplectic and the orthogonal complement of $V$. In fact, we have the following result, the proof of which we postpone.

Lemma 2.1. Let $V$ be an element in $G_{h}(n, m)$. Then, for all $u \in V$, we have

$$
\omega\left(\pi_{V^{\perp}}(v), u\right)=\omega\left(\pi_{J V}(v), u\right),
$$

where $J=\left(\begin{array}{cc}0 & I_{n} \\ -I_{n} & 0\end{array}\right)$.

In order to estimate the expression in (2.1) from above, the subsequent propositions are useful.

Proposition 2.2. Let $V \in G_{h}(n, m)$. For $0<s<1, c \in \mathbb{R}$ and $\bar{v} \in J V$ with $|\bar{v}|=1$, we have

$$
\int_{V \cap B(0, R)}|c+4 \omega(\bar{v}, u)|^{-s} \mathrm{~d} \mathcal{H}^{m}(u) \lesssim_{R} 1 .
$$


Proposition 2.3. For $0<s<m$ and all $z \in \mathbb{R}^{2 n} \backslash\{0\}$, we have

$$
\int_{G_{h}(n, m)}\left|\pi_{J V}(z)\right|^{-s} \mathrm{~d} \mu_{n, m}(V) \lesssim|z|^{-s} .
$$

Assuming the validity of these propositions, we demonstrate how to conclude the proof of Theorem 0.2. Proposition 2.3 shows that for a given $v$ with $|v|=1$, the set of elements $V \in G_{h}(n, m)$ where $\pi_{J V}(v)$ vanishes, is of zero measure, thus

$$
I \lesssim \int_{E}|z-\zeta|^{-s / 2} \int_{V \cap B(0, R)}\left|f(V)+4 \omega\left(\pi_{J V}(v), u\right)\right|^{-s / 2} \mathrm{~d} \mathcal{H}^{m}(u) \mathrm{d} \mu_{n, m}(V)
$$

with

$$
E:=\left\{V \in G_{h}(n, m): \pi_{J V}(v) \neq 0\right\} .
$$

It is therefore sufficient to estimate

$$
I(V):=|z-\zeta|^{-s / 2}\left|\pi_{J V}(v)\right|^{-s / 2} \int_{V \cap B(0, R)}\left|\frac{f(V)}{\left|\pi_{J V}(v)\right|}+4 \omega(\bar{v}, u)\right|^{-s / 2} \mathrm{~d} \mathcal{H}^{m}(u)
$$

for $V \in E$. Here, $\bar{v}=\frac{\pi_{J V}(v)}{\left|\pi_{J V}(v)\right|}$.

Similarly as in $[1,2]$, we distinguish several cases that can occur for a pair $(p, q) \in B$.

First, assume $|z-\zeta|^{2} \geq|t-\tau-2 \omega(z, \zeta)|$. Then,

$$
|z-\zeta|^{-s / 2} \simeq d_{H}(p, q)^{-s / 2} \lesssim d_{H}(p, q)^{-s},
$$

where the last inequality is due to the fact that $p$ and $q$ lie in a bounded set. In this case $^{1}$ we may simply apply Proposition 2.2 to deduce

$$
I(V) \lesssim|z-\zeta|^{-s / 2}\left|\pi_{J V}(v)\right|^{-s / 2}, \quad \text { for all } V \in E .
$$

Hence,

$$
\begin{aligned}
I & \lesssim \int_{E} I(V) \mathrm{d} \mu_{n, m}(V) \lesssim|z-\zeta|^{-s / 2} \int_{E}\left|\pi_{J V}(v)\right|^{-s / 2} \mathrm{~d} \mu_{n, m}(V) \\
& \lesssim|z-\zeta|^{-s / 2} \lesssim d_{H}(p, q)^{-s}
\end{aligned}
$$

by Proposition 2.3 for $s / 2 \leq m$.

Second, assume $|z-\zeta|^{2}<|t-\tau-2 \omega(z, \zeta)|$. In this case $|z-\zeta|$ can be much smaller than $d_{H}(p, q)$ and the above strategy does not yield a good enough estimate in general. A different approach is needed. We write $E=E_{1} \cup E_{2}$, where

$$
E_{1}:=\{V \in E:|f(V)| \geq 8 R\} \quad \text { and } \quad E_{2}:=\{V \in E:|f(V)|<8 R\} .
$$

${ }^{1}$ In higher dimensional Heisenberg groups, $\mathbb{H}^{n}$ with $n>1$, one can use the easier estimate $I \leq \int_{G_{h}(n, m)}\left|\pi_{V^{\perp}}(z-\zeta)\right|^{-s} \mathrm{~d} \mu_{n, m}$ for $s<2 n-m$. In the first Heisenberg group however, this works only for $s<1$, whereas the claim is formulated for $s<2$. 
Our goal is to estimate

$$
I \lesssim \int_{E_{1}} I(V) \mathrm{d} \mu_{n, m}(V)+\int_{E_{2}} I(V) \mathrm{d} \mu_{n, m}(V)
$$

from above by a constant multiple of $d_{H}(p, q)^{-s}$, for which purpose we will consider the two integrals separately.

We start with the integral over $E_{1}$. For $V \in E_{1}$, we have

$$
\begin{aligned}
\left|\frac{f(V)}{\left|\pi_{J V}(v)\right|}+4 \omega(\bar{v}, u)\right| & \geq\left|\frac{f(V)}{\left|\pi_{J V}(v)\right|}\right|-4|\omega(\bar{v}, u)| \\
& \geq\left|\frac{f(V)}{\left|\pi_{J V}(v)\right|}\right|-4 R \geq \frac{1}{2}\left|\frac{f(V)}{\left|\pi_{J V}(v)\right|}\right|
\end{aligned}
$$

for all $u \in B(0, R) \cap V$. Therefore,

$$
\begin{aligned}
I(V) & \lesssim|z-\zeta|^{-s / 2}\left|\pi_{J V}(v)\right|^{-s / 2}\left|\frac{f(V)}{\left|\pi_{J V}(v)\right|}\right|^{-s / 2} \mathcal{H}^{m}(B(0, R)) \\
& \simeq_{R}|z-\zeta|^{-s / 2}|f(V)|^{-s / 2}
\end{aligned}
$$

For $V \in E_{1}$, the expression on the right is comparable to $|t-\tau-2 \omega(z, \zeta)|^{-s / 2} \simeq$ $d_{H}(p, q)^{-s}$. Indeed,

$$
\begin{aligned}
\left|\frac{t-\tau-2 \omega(z, \zeta)}{|z-\zeta||z+\zeta|}\right|-\frac{|f(V)|}{2} & \leq\left|\frac{t-\tau-2 \omega(z, \zeta)}{|z-\zeta||z+\zeta|}\right|-2 \leq|f(V)| \\
& \leq\left|\frac{t-\tau-2 \omega(z, \zeta)}{|z-\zeta||z+\zeta|}\right|+2 \\
& \leq\left|\frac{t-\tau-2 \omega(z, \zeta)}{|z-\zeta||z+\zeta|}\right|+\frac{|f(V)|}{2}
\end{aligned}
$$

shows that

$$
|f(V)| \simeq\left|\frac{t-\tau-2 \omega(z, \zeta)}{|z-\zeta||z+\zeta|}\right| \simeq\left|\frac{t-\tau-2 \omega(z, \zeta)}{|z-\zeta|}\right|
$$

We conclude that

$$
\int_{E_{1}} I(V) \mathrm{d} \mu_{n, m}(V) \lesssim_{R} d_{H}(p, q)^{-s} .
$$

We continue with the integral over $E_{2}$. Assume that $E_{2}$ is non-empty, else nothing is to prove. For this part, the restriction on the range of $s$ is relevant. As in the first case which we have considered for $(p, q)$, we find by applying Proposition 2.2 and Proposition 2.3 that

$$
\int_{E_{2}} I(V) \mathrm{d} \mu_{n, m}(V) \lesssim|z-\zeta|^{-s / 2}
$$


The mere fact that $E_{2}$ contains at least one element $V$, gives information on the pair $(p, q)$ and thus on $|z-\zeta|$. Indeed,

$$
8 R>|f(V)| \geq\left|\frac{t-\tau-2 \omega(z, \zeta)}{|z-\zeta||z+\zeta|}\right|-2
$$

and hence

$$
(8 R+2)|z+\zeta||z-\zeta| \geq|t-\tau-2 \omega(z, \zeta)| .
$$

Thus, (2.4) yields

$$
\int_{E_{2}} I(V) \mathrm{d} \mu_{n, m}(V) \lesssim|t-\tau-2 \omega(z, \zeta)|^{-s / 2} \simeq d_{H}(p, q)^{-s}
$$

Together, (2.3) and (2.5) give

$$
I \lesssim d_{H}(p, q)^{-s}
$$

As we have considered all possible configurations of $(p, q)$, this shows

$$
\begin{aligned}
\int_{G_{h}(n, m)} \int_{V \cap B(0, R)} I_{s}\left(\mu_{V, u}\right) \mathrm{d} \mathcal{H}^{m}(u) \mathrm{d} \mu_{n, m}(V) & \lesssim \int_{B} \int_{B} d_{H}(p, q)^{-s} \mathrm{~d} \mu(p) \mathrm{d} \mu(q) \\
& =I_{s}(\mu)<\infty,
\end{aligned}
$$

and thus concludes the proof of the Theorem 0.2, up to the verification of Lemma 2.1, Proposition 2.2 and Proposition 2.3, which has been postponed.

Proof of Lemma 2.1. If $V$ and $V^{\perp}$ are orthogonal, then so are $J V$ and $J V^{\perp}$. Thus we can write

$$
\pi_{V^{\perp}}(v)=\pi_{J V}\left(\pi_{V^{\perp}}(v)\right)+\pi_{J V^{\perp}}\left(\pi_{V^{\perp}}(v)\right) .
$$

From the fact that $V$ and $V^{\perp}$ are orthogonal it follows

$$
\omega\left(\pi_{J V^{\perp}}(w), u\right)=0 \quad \text { for all } u \in V, w \in \mathbb{R}^{2 n},
$$

and thus

$$
\omega\left(\pi_{V^{\perp}}(v), u\right)=\omega\left(\pi_{J V}\left(\pi_{V^{\perp}}(v)\right), u\right) \quad \text { for all } u \in V .
$$

Since $V$ is isotropic, one has $J V \subseteq V^{\perp}$ and hence

$$
\pi_{J V}\left(\pi_{V^{\perp}}(v)\right)=\pi_{J V}(v)
$$

and (2.2) holds as desired. 
Proof of Proposition 2.2. First, if $|c| \geq 8 R$, then $|c+4 \omega(\bar{v}, u)| \geq|c| / 2$ for all $u \in B(0, R)$ and $\bar{v}$ of length 1 , and so

$$
\int_{V \cap B(0, R)}|c+4 \omega(\bar{v}, u)|^{-s} \mathrm{~d} \mathcal{H}^{m}(u) \lesssim_{R}|c|^{-s} \lesssim_{R} 1
$$

for all $s>0$. Second, we consider the case $|c|<8 R$. This can be dealt with by sub-level set estimates [6] or by a direct computation, which we show here. Given $V \in G_{h}(n, m)$, we may choose a basis $\left\{e_{1}, \ldots, e_{n}, f_{1}, \ldots, f_{n}\right\}$ for $\mathbb{R}^{2 n}$ so that $u=\sum_{i=1}^{m} u_{i} e_{i}, \bar{v}=\sum_{i=1}^{m} v_{i} f_{i}$ with $\sum_{i=1}^{m} v_{i}^{2}=1$, and

$$
F(u):=c+4 \omega(\bar{v}, u)=c+4 \sum_{i=1}^{m} v_{i} u_{i} .
$$

Without loss of generality $\left|v_{1}\right| \geq 1 / m$. Then

$$
\begin{aligned}
& \int_{V \cap B(0, R)}|F(u)|^{-s} \mathrm{~d} \mathcal{H}^{m}(u) \\
\leq & \int_{-R}^{R} \ldots \int_{-R}^{R}\left(\int_{-R}^{R}\left|c\left(u_{2}, \ldots, u_{m}\right)+4 v_{1} u_{1}\right|^{-s} \mathrm{~d} u_{1}\right) \mathrm{d} u_{2} \ldots \mathrm{d} u_{m},
\end{aligned}
$$

where $c\left(u_{2}, \ldots, u_{m}\right)=c+4 \sum_{i=2}^{m} v_{i} u_{i}$. Thus

$$
\left|c\left(u_{2}, \ldots, u_{m}\right)\right| \leq 8 R+4 R(m-1)=4 R(m+1)
$$

and then

$$
\begin{aligned}
\int_{-R}^{R}\left|c\left(u_{2}, \ldots, u_{m}\right)+4 v_{1} u_{1}\right|^{-s} \mathrm{~d} u_{1} & =\frac{1}{4 v_{1}} \int_{-c\left(u_{2}, \ldots, u_{m}\right)-4 R v_{1}}^{c\left(u_{2}, \ldots, u_{m}\right)+4 R v_{1}}|x|^{-s} d x \\
& \leq \frac{m}{4} \int_{-4 R(m+2)}^{4 R(m+2)}|x|^{-s} d x \lesssim_{R} 1
\end{aligned}
$$

since $s<1$. This yields

$$
\begin{aligned}
& \int_{V \cap B(0, R)}|F(u)|^{-s} \mathrm{~d} \mathcal{H}^{m}(u) \\
\leq & \int_{-R}^{R} \ldots \int_{-R}^{R}\left|c\left(u_{2}, \ldots, u_{m}\right)+4 v_{1} u_{1}\right|^{-s} \mathrm{~d} u_{1} \ldots \mathrm{d} u_{m} \lesssim_{R} 1
\end{aligned}
$$

as desired.

Proof of Proposition 2.3. We recall from [2] that

$$
\int_{G_{h}(n, m)}\left|\pi_{V}(z)\right|^{-s} \mathrm{~d} \mu_{n, m}(V) \lesssim|z|^{-s}
$$


for $s<m$ and $z \in \mathbb{R}^{2 n} \backslash\{0\}$. The nice behaviour of scalar products under the map $J: \mathbb{R}^{2 n} \rightarrow \mathbb{R}^{2 n}$ allows us to exploit this fact. Indeed, $J$ is an isometry. Moreover, if $\left\{e_{1}, \ldots, e_{m}\right\}$ is an orthonormal basis for $V$, then $\left\{J e_{1}, \ldots, J e_{m}\right\}$ is an orthonormal basis for $J V$, and we find

$$
\left|\pi_{J V}(z)\right|=\left(\sum_{i=1}^{m}\left(\left\langle z, J e_{i}\right\rangle\right)^{2}\right)^{\frac{1}{2}}=\left(\sum_{i=1}^{m}\left(\left\langle J z, e_{i}\right\rangle\right)^{2}\right)^{\frac{1}{2}}=\left|\pi_{V}(J z)\right|
$$

since $\langle v, J V\rangle=\left\langle J^{T} v, w\right\rangle=\langle-J v, w\rangle$ for all $v, w \in \mathbb{R}^{2 n}$. Thus, by the estimate (2.6), one obtains

$$
\int_{G_{h}(n, m)}\left|\pi_{J V}(z)\right|^{-s} \mathrm{~d} \mu_{n, m}(V)=\int_{G_{h}(n, m)}\left|\pi_{V}(J z)\right|^{-s} \mathrm{~d} \mu_{n, m}(V) \lesssim|J z|^{-s}=|z|^{-s},
$$

which concludes the proof of the proposition.

\section{Proof of Theorem 0.3}

The proof of Theorem 0.3 illustrates that the method developed by T. Orponen in [17] has applications beyond orthogonal projections in Euclidean spaces and, in fact, we consider it a particular strength of this method that it can be applied also in a setting where other techniques from Euclidean spaces such as dimension estimation by Fourier analysis - as used for instance in [16] - are not available. For the convenience of the reader, we provide the full proof of Theorem 0.3 , including the arguments which go along the lines of the reasoning in [17], but we will put special emphasis on those parts where the geometry of the Heisenberg group becomes relevant.

Proof of Theorem 0.3. We may assume that $B$ is bounded and contained in a ball $B(0, R)$. We fix $1<\sigma<1+\alpha(s)<s$, where $\alpha(s)$ is as in $(0.4)$, and make the counter assumption that

$$
\operatorname{dim}_{\mathrm{H}} B_{\theta}<\sigma \quad \text { for } \theta \in E \text {, }
$$

where $E \subset[0, \pi)$ has positive length. Let us sketch how this assumption can be used. From (3.1), we deduce that at arbitrary small scales $\delta$, the left-translates of the $c \delta^{1 / 2}$-neighborhood $\mathcal{C}$ of the $(x, y)$-plane have large $\mu$ mass with high probability. Here $\mu$ is a Frostman probability measure with exponent $s$ that we choose at the beginning. Indeed, observe that the left translate of the $(x, y)$-plane comprises left cosets of horizontal subgroups and if dimension drops unnaturally in one direction, it is to be expected that a lot of mass is concentrated in tubes around cosets which correspond to this direction. The fact that $\mu(p * \mathcal{C})$ is large for many $p$ 's, implies that there must exist well separated points $p_{1}$ and $p_{2}$ so that $\mu\left(\left(p_{1} * \mathcal{C}^{\prime}\right) \cap\left(p_{2} * \mathcal{C}^{\prime}\right)\right)$ is large as well, where $\mathcal{C}^{\prime}$ denotes a $\delta^{1 / 4}$-neighborhood of the horizontal plane. 
However, under the given assumptions, the intersection $\left(p_{1} * \mathcal{C}^{\prime}\right) \cap\left(p_{2} * \mathcal{C}^{\prime}\right) \cap B(0, R)$ will be contained in a small neighborhood of a line. A line in the Heisenberg group is at most two-dimensional and the fact that $\mu$ is a measure with exponent $s>2$ for the growth bound then shows that $\mu\left(\left(p_{1} * \mathcal{C}\right) \cap\left(p_{2} * \mathcal{C}\right)\right)$ cannot be too large, which leads to a contradiction and thus proves $\operatorname{dim}_{\mathrm{H}} B_{\theta} \geq \sigma$ for almost every $\theta$. Letting $\sigma$ tend to $1+\alpha(s)$ then concludes the proof of the theorem.

We now discuss the proof in detail. Our first goal is analogous to the strategy in [5, page 222], which also marks the start of the proof in [17]. By assumption,

$$
\sup _{\delta_{0}>0} \mathcal{H}_{H, \delta_{0}}^{\sigma}\left(B_{\theta}\right)=\mathcal{H}_{H}^{\sigma}\left(B_{\theta}\right)=0
$$

for every $\theta \in E$. Fix $\delta_{0}>0$. It follows from (3.2) that for every $\theta \in E$ we may cover the projection $B_{\theta}$ with a collection of Korányi balls

$$
\bigcup_{k \in \mathbb{N}: 2^{-k}<\delta_{0}} \widetilde{\mathcal{G}}_{k, \theta},
$$

where every $\widetilde{\mathcal{G}}_{k, \theta}$ is a family of $\lesssim 2^{k \sigma}$ balls of diameter $2^{-k}$. The collection $\widetilde{\mathcal{G}}_{k, \theta}$ is not disjoint to begin with, but we have the following lemma.

Lemma 3.1. Assume that (3.2) holds. Then, for any $\delta_{0}>0$, there exist collections of Borel sets $\mathcal{G}_{k, \theta}, 2^{-k}<\delta_{0}$, with the properties that

(1) the sets in $\mathcal{G}_{k, \theta}$ are disjoint,

(2) they have diameter $\leq 2^{-k}$,

(3) there are no more than $\lesssim 2^{k \sigma}$ sets in $\mathcal{G}_{k, \theta}$, and

$$
B_{\theta} \subset \bigcup_{k: 2^{-k}<\delta_{0}} \bigcup_{B_{i} \in \mathcal{G}_{k, \theta}} B_{i}
$$

This corresponds to [17, Lemma 3.2], yet, we choose a different approach for the proof and as a consequence, get a collection $\mathcal{G}_{k, \theta}$ which does not necessarily consist of balls. For the further application it will be enough to know that it comprises sets of diameter at most $2^{-k}$.

Proof. We start with the collection $\widetilde{\mathcal{G}}_{k, \theta}$ that we have found above. It consists of balls

$$
B\left(p_{1}, 2^{-k-1}\right), \ldots, B\left(p_{N}, 2^{-k-1}\right) \quad \text { with } N \lesssim 2^{k \sigma} .
$$

Set

$$
B_{1}:=B\left(p_{1}, 2^{-k-1}\right), \quad B_{n}:=B\left(p_{n}, 2^{-k-1}\right) \backslash \bigcup_{i=1}^{n-1} B_{i} \text { for } n \geq 2 .
$$

Without loss of generality, assume that $\mathcal{H}_{H}^{s}(B)>0$, and choose a Frostman probability measure $\mu$ supported on $B$ and satisfying $\mu(B(p, r)) \lesssim r^{s}$ for $p \in \mathbb{H}^{1}$ and $r>0$. 
Supposing (3.2), we choose for a given $\theta \in E$ and $\delta_{0}>0$ collections $\mathcal{G}_{k, \theta}$ as in Lemma 3.1. Then,

$$
1=\mu(B)=\mu\left(P_{\mathbb{V}_{\theta}^{-1}}^{-1}\left(B_{\theta}\right)\right) \leq \sum_{k: 2^{-k}<\delta_{0}} \mu\left(P_{\mathbb{V}_{\theta}^{-1}}^{-1}\left(\bigcup \mathcal{G}_{k, \theta}\right)\right) .
$$

As in [17], we find $k \in \mathbb{N}$ with $2^{-k}<\delta_{0}$ such that

$$
\mu\left(P_{\mathbb{V}_{\theta}^{\perp}}^{-1}\left(\cup \mathcal{G}_{k, \theta}\right)\right) \gtrsim k^{-2}
$$

for all $\theta$ in a set $E_{k} \subset E$ with $\left|E_{k}\right| \gtrsim k^{-2}$. Having fixed such $k$, we denote

$$
\delta:=2^{-k}<\delta_{0}, \quad \mathcal{G}_{\theta}:=\mathcal{G}_{k, \theta}, \quad E_{\delta}:=E_{k} .
$$

Definition 3.2. Let $\theta \in[0, \pi)$. For points $p, q \in \mathbb{H}^{1}$, we write

$$
p \sim_{\theta} q \quad \text { iff } \quad p, q \in P_{\mathbb{V}_{\theta}^{\perp}}^{-1}\left(B_{i}\right) \text { for some } B_{i} \in \mathcal{G}_{\theta},
$$

and we define the $\delta$-energy

$$
\mathcal{E}_{\delta}:=\int_{E_{\delta}} \mu \times \mu\left(\left\{(p, q): p \sim_{\theta} q\right\}\right) \mathrm{d} \theta .
$$

Notice that $p \sim_{\theta} q$ implies that $d_{H}\left(P_{\mathbb{V}_{\theta}^{\perp}}(p), P_{\mathbb{V}_{\theta}^{\perp}}(q)\right) \leq \delta$.

Our goal is to find an upper and a lower bound for $\mathcal{E}_{\delta}$. Repeating the same procedure for arbitrarily small $\delta_{0}>0$, this leads to an inequality valid for arbitrarily small $\delta$ - which turns out to be possible only if small neighbourhoods of left translates of the $(x, y)$-plane have large mass with high probability. This is intuitively plausible, as we are working at scales where in many directions tubes around left cosets of horizontal subgroups have large mass.

The lower bound for $\mathcal{E}_{\delta}$ is obtained completely analogous as in [17] using the assumptions that for $\theta \in E_{\delta}$ the union $\bigcup_{i=1}^{N} T_{i}$ with $T_{i}:=P_{\mathbb{V}_{\theta}^{-1}}^{-1}\left(B_{i}\right)$ is of large $\mu$ measure, the number $N$ is bounded from above, and $\left|E_{\delta}\right|$ cannot be too small. More precisely, for $\theta \in E_{\delta}$, condition (3.3) guarantees the existence of $T_{1}, \ldots, T_{N}$ with $\mu\left(\bigcup T_{j}\right) \gtrsim(\log 1 / \delta)^{-2}$, for $j=1, \ldots, N$ and $N \lesssim \delta^{-\sigma}$. Notice that for each $j$, we have

$$
T_{j} \times T_{j} \subset\left\{(p, q): p \sim_{\theta} q\right\}
$$

and the tubes are pairwise disjoint. Using this information and the Cauchy-Schwarz inequality, we obtain

$$
\begin{aligned}
\mu \times \mu\left(\left\{(p, q): p \sim_{\theta} q\right\}\right) & \gtrsim \sum_{j=1}^{N}\left(\mu\left(T_{j}\right)\right)^{2} \geq \frac{1}{N}\left(\sum_{j=1}^{N} \mu\left(T_{j}\right)\right)^{2} \gtrsim \delta^{\sigma} \cdot \mu\left(\bigcup_{j=1}^{N} T_{j}\right)^{2} \\
& \gtrsim \delta^{\sigma} \cdot\left(\log \left(\frac{1}{\delta}\right)\right)^{-4} .
\end{aligned}
$$


Now we integrate with respect to $\theta$ over $E_{\delta}$ and exploit the estimate $\left|E_{\delta}\right| \gtrsim$ $(\log (1 / \delta))^{-2}$, which yields

$$
\delta^{\sigma} \cdot\left(\log \left(\frac{1}{\delta}\right)\right)^{-6} \lesssim \mathcal{E}_{\delta}
$$

To obtain a suitable upper bound for $\mathcal{E}_{\delta}$, we first note that

$$
\mathcal{E}_{\delta}=\iint\left|\left\{\theta \in E_{\delta}: p \sim_{\theta} q\right\}\right| \mathrm{d} \mu(p) \mathrm{d} \mu(q) .
$$

The integrand will be zero for $p$ lying outside the sets $q * \mathcal{C}$ introduced below. The definition of $q * \mathcal{C}$ is motivated by the following lemma.

Lemma 3.3. For every $R>0$ there exists a finite constant $c=c(R)>0$ such that

$$
P_{\mathbb{V}_{\theta}^{\perp}}^{-1}\left(B\left(p_{\mathbb{V}_{\theta}^{\perp}}, \delta\right)\right) \cap B(0, R) \subseteq N_{H}\left(p * \mathbb{V}_{\theta}, c \delta^{\frac{1}{2}}\right) \cap B(0, R)
$$

for all $p_{\mathbb{V}_{\theta}^{\perp}} \in \mathbb{V}_{\theta}^{\perp}$ and $p \in \mathbb{H}^{1}$ with $P_{\mathbb{V}_{\theta}^{\perp}}(p)=p_{\mathbb{V}_{\frac{\theta}{\theta}}}$.

Proof. Let $q=(\zeta, \tau)$ be an arbitrary point in $P_{\mathbb{V}_{\theta}^{\perp}}^{-1}\left(B\left(p_{\mathbb{V}_{\theta}^{\perp}}, \delta\right)\right) \cap B(0, R)$. Thus in particular

$$
|\zeta| \leq R \quad \text { and } \quad d_{H}\left(q_{\mathbb{V}_{\theta}^{\perp}}, p_{\mathbb{V}_{\theta}^{\perp}}\right) \leq \delta .
$$

Denoting $p_{\mathbb{V}_{\theta}^{\perp}}=\left(\pi_{V_{\theta}^{\perp}}(z), t^{\prime}\right)$ and $q_{\mathbb{V}_{\theta}^{\perp}}=\left(\pi_{V_{\theta}^{\perp}}(\zeta), \tau^{\prime}\right)$, the last inequality gives

$$
\left|\pi_{V_{\theta}^{\perp}}(\zeta-z)\right| \leq \delta \quad \text { and } \quad\left|\tau^{\prime}-t^{\prime}\right| \leq \delta^{2} .
$$

Then

$$
\begin{aligned}
d_{H}\left(q_{\mathbb{V}_{\theta}^{\perp}} q_{\mathbb{V}_{\theta}}, p_{\mathbb{V}_{\theta}^{\perp}} q_{\mathbb{V}_{\theta}}\right) & =\sqrt[4]{\left|\pi_{V_{\theta}^{\perp}}(\zeta-z)\right|^{4}+\left(\tau^{\prime}-t^{\prime}+2 \operatorname{Im}\left(\pi_{V_{\theta}^{\perp}}(\zeta-z) \overline{\pi_{V_{\theta}}(\zeta)}\right)\right)^{2}} \\
& \leq \sqrt[4]{\delta^{4}+\left(\delta^{2}+2|\zeta| \delta\right)^{2}} \\
& \leq c(R) \delta^{\frac{1}{2}}
\end{aligned}
$$

Thus,

$$
P_{\mathbb{V}_{\theta}^{\perp}}^{-1}\left(B\left(p_{\mathbb{V}_{\theta}^{\perp}}, \delta\right)\right) \cap B(0, R) \subseteq N_{H}\left(p_{\mathbb{V}_{\theta}^{\perp}} * V_{\theta}, c \delta^{\frac{1}{2}}\right)
$$

and the claimed inclusion follows since $p_{\mathbb{V}_{\theta}^{\perp}} * \mathbb{V}_{\theta}=p * \mathbb{V}_{\theta}$ for all $p \in \mathbb{H}^{1}$ with $P_{\mathbb{V}_{\theta}^{\perp}}(p)=p_{\mathbb{V}_{\theta}^{\perp}}$. 
This motivates the definition

$$
\mathcal{C}:=\bigcup_{\theta \in[0, \pi)} N_{H}\left(\mathbb{V}_{\theta}, c \delta^{1 / 2}\right)
$$

Notice that

$$
p * \mathcal{C}=\bigcup_{\theta} N_{H}\left(p * \mathbb{V}_{\theta}, c \delta^{1 / 2}\right)=N_{H}\left(\bigcup_{\theta} p * \mathbb{V}_{\theta}, c \delta^{1 / 2}\right)=N_{H}\left(H_{p}, c \delta^{1 / 2}\right),
$$

where by $H_{p_{0}}$, we denote the left translate of the $(x, y)$-plane by the group element $p_{0}=\left(x_{0}, y_{0}, t_{0}\right)$, that is,

$$
\begin{aligned}
H_{p_{0}} & =\left(x_{0}, y_{0}, t_{0}\right) *\left\{\left(x^{\prime}, y^{\prime}, 0\right):\left(x^{\prime}, y^{\prime}\right) \in \mathbb{R}^{2}\right\} \\
& =\left\{\left(x_{0}+x^{\prime}, y_{0}+y^{\prime}, t_{0}-2 x_{0} y^{\prime}+2 y_{0} x^{\prime}\right):\left(x^{\prime}, y^{\prime}\right) \in \mathbb{R}^{2}\right\} \\
& =\left\{(x, y, t):-2 y_{0} x+2 x_{0} y+t-t_{0}=0\right\} .
\end{aligned}
$$

We note that the set $(p * \mathcal{C}) \cap B(0, R)$ is contained in the Euclidean neighbourhood $N_{E}\left(H_{p},\left(c / c_{1}\right) \delta^{1 / 2}\right) \cap B(0, R)$ of the plane $H_{p}$.

Remark 3.4. In the Euclidean setting, one has $\pi_{\theta}^{-1}\left(B\left(\pi_{\theta}(p), \delta\right)\right)=p+\pi_{\theta}^{-1}(B(0, \delta))$, but it is not true that $p * P_{\mathbb{V}_{\theta}^{\perp}}^{-1}(B(0, \delta))=P_{\mathbb{V}_{\theta}^{\perp}}^{-1}\left(B\left(P_{\mathbb{V}_{\theta}^{\perp}}(p), \delta\right)\right)$; this is why we enlarge $\bigcup P_{\mathbb{V}_{\theta}^{\perp}}^{-1}\left(B\left(P_{\mathbb{V}_{\theta}^{\perp}}(p), \delta\right)\right)$, and consider the $c \delta^{1 / 2}$ neighbourhood of $p * H_{p}$ instead. This works fine as long as we are only considering the intersections of the respective sets with some bounded neighbourhood of the the origin.

Now, if two points $p, q \in \operatorname{spt} \mu \subset B(0, R)$ have the property $p \notin q * \mathcal{C}$, then for all $\theta \in[0, \pi)$ we have $p \notin N_{H}\left(q * \mathbb{V}_{\theta}, c \delta^{1 / 2}\right)$ and thus in particular $p \notin P_{\mathbb{V}_{\theta}^{\perp}}^{-1}\left(B\left(q_{\mathbb{V}_{\theta}^{\perp}}, \delta\right)\right)$ so that

$$
d_{H}\left(P_{\mathbb{V}_{\theta}^{\perp}}(p), P_{\mathbb{V}_{\theta}^{\perp}}(q)\right)>\delta
$$

and then $p \nsim_{\theta} q$ since the sets $B_{i}$ in $\mathcal{G}_{\theta}$ are of diameter at most $\delta$. Thus,

$$
\mathcal{E}_{\delta}=\iint_{q * \mathcal{C}}\left|\left\{\theta \in E_{\delta}: p \sim_{\theta} q\right\}\right| \mathrm{d} \mu(p) \mathrm{d} \mu(q) .
$$

In order to bound the integrand from above, we make use of a sub-level set estimate which appears implicitly already in [2].

Lemma 3.5. We have

$$
\left|\left\{\theta \in[0, \pi): d_{H}\left(P_{\mathbb{V}_{\theta}^{\perp}}(p), P_{\mathbb{V}_{\theta}^{\perp}}(q)\right) \leq \delta\right\}\right| \lesssim \frac{\delta}{d_{H}(p, q)}
$$

for all distinct points $p, q \in \mathbb{H}^{1}$. 
Proof. We consider two cases associated to the sets

$$
A_{1}=\left\{(p, q):|z-\zeta|^{4} \geq(t-\tau-2 \omega(\zeta, z))^{2}\right\}
$$

and

$$
A_{2}=\left\{(p, q):|z-\zeta|^{4}<(t-\tau-2 \omega(\zeta, z))^{2}\right\}
$$

If $(p, q) \in A_{1}$, we have by the sub-level set estimate for usual orthogonal projections,

$$
\begin{aligned}
\left|\left\{\theta \in[0, \pi): d_{H}\left(P_{\mathbb{V}_{\theta}^{\perp}}(p), P_{\mathbb{V}_{\theta}^{\perp}}(q)\right) \leq \delta\right\}\right| & \leq\left|\left\{\theta \in[0, \pi):\left|\pi_{\mathbb{V}_{\theta}^{\perp}}(z-\zeta)\right| \leq \delta\right\}\right| \\
& \leq \frac{\delta}{|z-\zeta|} \lesssim \frac{\delta}{d_{H}(p, q)} .
\end{aligned}
$$

If $(p, q) \in A_{2}$, we will further distinguish several cases. If $z= \pm \zeta$, we have

$$
d_{H}\left(P_{\mathbb{V}_{\theta}^{\perp}}(p), P_{\mathbb{V}_{\theta}^{\perp}}(q)\right) \geq|t-\tau|^{1 / 2}=d_{H}(p, q)
$$

and so

$$
\begin{aligned}
\left|\left\{\theta \in[0, \pi): d_{H}\left(P_{\mathbb{V}_{\theta}^{\perp}}(p), P_{\mathbb{V}_{\theta}^{\perp}}(q)\right) \leq \delta\right\}\right| & \leq\left|\left\{\theta \in[0, \pi): d_{H}(p, q) \leq \delta\right\}\right| \\
& = \begin{cases}0 & d_{H}(p, q)>\delta \\
\pi & d_{H}(p, q) \leq \delta\end{cases} \\
& \lesssim \frac{\delta}{d_{H}(p, q)} .
\end{aligned}
$$

Denoting

$$
a:=\frac{t-\tau-2 \omega(\zeta, z)}{|z+\zeta||z-\zeta|}, \quad v=\frac{z-\zeta}{|z-\zeta|}, \quad w=\frac{z+\zeta}{|z+\zeta|},
$$

if $z \neq \pm \zeta$, we estimate

$$
\begin{aligned}
d_{H}\left(P_{\mathbb{V}_{\theta}^{\perp}}(p), P_{\mathbb{V}_{\theta}^{\perp}}(q)\right) & \geq(|z+\zeta||z-\zeta|)^{1 / 2}\left|a-2 \omega\left(v, \pi_{V_{\theta}}(w)\right)\right|^{1 / 2} \\
& \gtrsim \begin{cases}|z+\zeta|^{1 / 2}|z-\zeta|^{1 / 2}|a| \simeq d_{H}(p, q) \text { if }|a| \geq 4 \\
d_{H}(p, q)\left|a-2 \omega\left(v, \pi_{V_{\theta}}(w)\right)\right|^{1 / 2} & \text { if }|a|<4,\end{cases}
\end{aligned}
$$

see [2]. The first case is trivial. In the second case, we arrive at

$$
\begin{aligned}
& \left|\left\{\theta \in[0, \pi): d_{H}\left(P_{\mathbb{V}_{\theta}^{\perp}}(p), P_{\mathbb{V}_{\theta}^{\perp}}(q)\right) \leq \delta\right\}\right| \\
& \leq\left|\left\{\theta:\left|a-2 \omega\left(v, \pi_{V_{\theta}}(w)\right)\right|^{1 / 2} \leq \frac{\delta}{d_{H}(p, q)}\right\}\right| .
\end{aligned}
$$

Consider the function

$$
F(\theta)=a-2 \omega\left(v, \pi_{V_{\theta}}(w)\right) \quad \text { with } \quad \pi_{V_{\theta}}(w)=\left(\cos \theta w_{1}+\sin \theta w_{2}\right)\left(\begin{array}{c}
\cos \theta \\
\sin \theta
\end{array}\right) .
$$


One finds

$$
F^{\prime}(\theta)=-2 \omega\left(v, \partial_{\theta} \pi_{V_{\theta}}(w)\right) \quad \text { and } \quad F^{\prime \prime}(\theta)=-2 \omega\left(v, \partial_{\theta}^{2} \pi_{V_{\theta}}(w)\right)
$$

and observes that $F^{\prime}$ and $F^{\prime \prime}$ cannot vanish at the same time. The desired result follows then from the sub-level set estimate in [6] and compactness arguments as shown in [2].

For the subsequent parts of the proof, set

$$
\tau:=\frac{1}{2}\left(\frac{(\sigma-1) s}{s-1}+\frac{s-2}{32 s}\right)
$$

and define the 'good' set

$$
G:=\left\{q \in \mathbb{H}^{1}: \mu(q * \mathcal{C}) \geq \delta^{\tau}\right\} .
$$

The parameter $\tau$ is chosen so that $\mu(G) \gtrsim \delta^{\tau}$, as we are going to see now. By Lemma 3.5 and the mass bound $\mu(B(p, r)) \lesssim r^{s}$ with $s>1$, we have

$$
\begin{aligned}
I_{G} & :=\int_{G} \int_{q * \mathcal{C}}\left|\left\{\theta \in E_{\delta}: p \sim_{\theta} q\right\}\right| \mathrm{d} \mu(p) \mathrm{d} \mu(q) \\
& \lesssim \delta \int_{G} \int \frac{1}{d_{H}(p, q)} \mathrm{d} \mu(p) \mathrm{d} \mu(q) \lesssim \delta \mu(G) .
\end{aligned}
$$

Analogously as in [17], we estimate the second summand in $\mathcal{E}_{\delta}$ by

$$
\begin{aligned}
I_{\mathbb{H}^{1} \backslash G}: & =\int_{\mathbb{H}^{1} \backslash G} \int_{q * \mathcal{C}}\left|\left\{\theta \in E_{\delta}: p \sim_{\theta} q\right\}\right| \mathrm{d} \mu(p) \mathrm{d} \mu(q) \\
\lesssim & \int_{\mathbb{H}^{1} \backslash G} \int_{B(y, 2 R \delta)} \mathrm{d} \mu(p) \mathrm{d} \mu(q) \\
& +\int_{\mathbb{H}^{1} \backslash G} \sum_{\delta \leq 2^{-j} \leq 1} \int_{(q * \mathcal{C}) \cap A_{j}(q)}\left|\left\{\theta \in[0, \pi): p \sim_{\theta} q\right\}\right| \mathrm{d} \mu(p) \mathrm{d} \mu(q) \\
\lesssim & \delta^{s}+\delta^{1+\tau(1-1 / s)} \cdot\left(\log \left(\frac{1}{\delta}\right)\right),
\end{aligned}
$$

using again Lemma 3.5, decomposition of the space in dyadic annuli $A_{j}(q):=$ $\left\{p \in \mathbb{H}^{1}: 2^{-j} \cdot 2 R \leq d_{H}(p, q) \leq 2^{-j+1} \cdot 2 R\right\}$ and the growth bound for $\mu$. More precisely, for $q \notin G$, we have by definition of $G$ and $A_{j}(q)$ that

$$
\mu\left((q * \mathcal{C}) \cap A_{j}(q)\right) \lesssim_{R} \min \left\{\delta^{\tau}, 2^{-j s}\right\}=\delta^{\tau(1-1 / s)} 2^{-j}
$$

Combining (3.4), (3.8) and (3.9) yields

$$
\delta^{\sigma} \cdot\left(\log \left(\frac{1}{\delta}\right)\right)^{-6} \lesssim \mathcal{E}_{\delta} \lesssim \delta \mu(G)+\delta^{s}+\delta^{1+\tau(1-1 / s)} \cdot\left(\log \left(\frac{1}{\delta}\right)\right) .
$$


Since $\sigma<1+\tau(1-1 / s)<1+\alpha(s)<s$, the only situation when the right hand side can dominate the left hand side is if

$$
\mu(G) \gtrsim \delta^{\sigma-1} \geq \delta^{\tau},
$$

that is, $q * \mathcal{C}$ has large $\mu$-measure with high probability. This fact can be used to find separated points $p_{1}, p_{2}$ so that the intersection $\left(p_{1} * \mathcal{C}^{\prime}\right) \cap\left(p_{2} * \mathcal{C}^{\prime}\right)$ is of large measure. Here

$$
\mathcal{C}^{\prime}:=\bigcup N_{H}\left(\mathbb{V}_{\theta}, c^{\prime} \delta^{1 / 4}\right)
$$

where the constant $c^{\prime}=c^{\prime}(R) \geq 0$ will be chosen below.

Lemma 3.6. There exists a constant $c^{\prime}=c^{\prime}(R) \geq 0$ such that

$$
\mathcal{C} \cap B(0, R) \subseteq \mathcal{C}^{\prime-1} \cap B(0, R) .
$$

Proof. We have

$$
\begin{aligned}
\mathcal{C} \cap B(0, R) & =\bigcup N_{H}\left(\mathbb{V}_{\theta}, c \delta^{\frac{1}{2}}\right) \cap B(0, R) \subseteq \mathcal{C}^{\prime-1} \cap B(0, R) \\
& =\left\{p^{-1}: p \in \mathcal{C}^{\prime}\right\} \cap B(0, R)
\end{aligned}
$$

for a constant $c^{\prime}=c^{\prime}(R) \geq 0$. Indeed, for

$$
p=\left((\rho+i \xi) e^{i \theta}, \tau\right) \quad \text { and } \quad q=\left(r e^{i \theta}, 0\right)
$$

we find

$$
d_{H}(p, q)=\sqrt[4]{\left((\rho-r)^{2}+\xi^{2}\right)^{2}+(\tau+2 r \xi)^{2}}
$$

and

$$
d_{H}\left(p^{-1}, q^{-1}\right)=\sqrt[4]{\left((\rho-r)^{2}+\xi^{2}\right)^{2}+(\tau+2 r \xi-2 \tau)^{2}} .
$$

For $p \in \mathcal{C} \cap B(0, R)$, we have $|\rho| \leq R$. Now if $d_{H}(p, q) \leq c \delta^{1 / 2}$ for some $q \in \mathbb{V}_{\theta}$, then $|\rho-r| \leq c \delta^{1 / 2},|\xi| \leq c \delta^{1 / 2}$ and $|\tau+2 r \xi| \leq c^{2} \delta$ and so $|\tau| \lesssim \delta^{1 / 2}$. Hence, by the above formula $d_{H}\left(p^{-1}, q^{-1}\right) \lesssim \delta^{\frac{1}{4}}$ and therefore $p \in \mathcal{C}^{\prime-1}$.

Proposition 3.7. Under the counter-assumption (3.1) and for the choice of $\mu$ as above, there exist $p_{1}, p_{2} \in B(0, R)$ with

$$
d_{H}\left(p_{1}, p_{2}\right)>2 \delta^{\kappa} \quad \text { and } \mu\left(\left(p_{1} * \mathcal{C}^{\prime}\right) \cap\left(p_{2} * \mathcal{C}^{\prime}\right)\right) \geq \delta^{\kappa},
$$

where

$$
\kappa:=2 \tau+\frac{s-2}{16 s} .
$$


Proof. We start by defining

$$
\begin{aligned}
A & :=\iint \mu\left(\left(p_{1} * \mathcal{C}^{\prime}\right) \cap\left(p_{2} * \mathcal{C}^{\prime}\right)\right) \mathrm{d} \mu\left(p_{1}\right) \mathrm{d} \mu\left(p_{2}\right) \\
& =\int \mu\left(\left\{p: q \in p * \mathcal{C}^{\prime}\right\}\right)^{2} \mathrm{~d} \mu(q)=\int \mu\left(q * \mathcal{C}^{\prime-1}\right)^{2} \mathrm{~d} \mu(q)
\end{aligned}
$$

where $\mathcal{C}^{\prime-1}:=\left\{p^{-1}: p \in \mathcal{C}^{\prime}\right\}$. By Lemma 3.6 and Hölder's inequality,

$$
A \geq\left(\int_{G} \mu(q * \mathcal{C}) \mathrm{d} \mu(q)\right)^{2} \gtrsim \delta^{4 \tau}
$$

Given a pair $\left(p_{1}, p_{2}\right)$, either one of the points is contained in the $2 \delta^{\kappa}$ ball centred at the other point, or they are well separated. If they are separated, either $\mu\left(\left(p_{1} *\right.\right.$ $\left.\left.\mathcal{C}^{\prime}\right) \cap\left(p_{2} * \mathcal{C}^{\prime}\right)\right)<\delta^{\kappa}$ or $\mu\left(\left(p_{1} * \mathcal{C}^{\prime}\right) \cap\left(p_{2} * \mathcal{C}^{\prime}\right)\right) \geq \delta^{\kappa}$. This leads us to the estimate

$$
\delta^{4 \tau} \lesssim A \lesssim 2^{s} \delta^{s \kappa}+\delta^{\kappa}+\mu \times \mu\left(A^{\prime}\right)
$$

where

$$
A^{\prime}=\left\{\left(p_{1}, p_{2}\right): d_{H}\left(p_{1}, p_{2}\right)>2 \delta^{\kappa}, \mu\left(\left(p_{1} * \mathcal{C}^{\prime}\right) \cap\left(p_{2} * \mathcal{C}^{\prime}\right)\right) \geq \delta^{\kappa}\right\} .
$$

If we now choose $\kappa$ as in (3.11), then $\kappa>4 \tau$ (and $\kappa<(s-2) / 8 s<1 / 8$, which will be used later) and thus we must have

$$
\mu \times \mu\left(A^{\prime}\right) \gtrsim \delta^{4 \tau}
$$

In particular, there exists $\left(p_{1}, p_{2}\right) \in \operatorname{spt} \mu \times \mu \subset B(0, R) \times B(0, R)$ with the desired properties.

The following proposition exhibits that the intersection $\left(p_{1} * \mathcal{C}^{\prime}\right) \cap\left(p_{2} * \mathcal{C}^{\prime}\right)$ for such a pair can in fact not be too large. This will eventually lead to a contradiction.

Proposition 3.8. There exists $\delta_{0}=\delta_{0}(R)>0$ such that whenever $\delta<\delta_{0}$ and $p, q$ are points in $B(0, R) \subset \mathbb{H}^{1}$ with $d_{H}(p, q)>2 \delta^{\kappa}$ then $\left(p * \mathcal{C}^{\prime}\right) \cap\left(q * \mathcal{C}^{\prime}\right) \cap B(0, R)$ is contained in the $c_{2} \delta^{\gamma}$ neighborhood (with respect to $d_{H}$ ) of a line, where $\gamma=$ $\frac{1}{16}-\frac{\kappa}{2}$.

Proof. The computations are easier if one of the two points is the origin. We will prove that $\mathcal{C}^{\prime} \cap\left(\left(p^{-1} q\right) * \mathcal{C}^{\prime}\right) \cap B(0,2 R)$ is either empty or contained in an appropriate neighbourhood of a line. The result then follows by left translation, since this is an isometry which preserves lines.

So let us assume from now on that $p=0$ and $q=(x, y, t)$ with $\|q\|_{H}=$ $\left(\left(x^{2}+y^{2}\right)^{2}+t^{2}\right)^{1 / 4}>2 \delta^{\kappa}$. From (1.1) it can be seen that $\left(p * \mathcal{C}^{\prime}\right) \cap B(0,2 R)$ and $\left(q * \mathcal{C}^{\prime}\right) \cap B(0,2 R)$ are contained in the Euclidean $\left(c^{\prime} / c_{1}\right) \delta^{1 / 4}$-neighbourhoods of 
planes. Information about the intersection of these neighbourhoods can be gained by looking at the normal vectors to the corresponding planes

$$
N_{p}=\left(\begin{array}{l}
0 \\
0 \\
1
\end{array}\right) \quad \text { and } \quad N_{q}=\frac{1}{\sqrt{4\left(x^{2}+y^{2}\right)+1}}\left(\begin{array}{c}
-2 y \\
2 x \\
1
\end{array}\right) .
$$

We first recall some facts about the intersection of tubes in $\mathbb{R}^{n}$. For $e \in S^{n-1}$ we write

$$
T_{e}^{\delta}(p):=\left\{q=p+\alpha e+v:|\alpha|<\frac{1}{2}, v \in e^{\perp},|v| \leq \delta\right\}
$$

for the $\delta$-tube of length one centred at $p \in \mathbb{R}^{n}$. Then the following result, which was used by A. Cordoba in his work on the Kakeya maximal function, holds.

Lemma 3.9. Given $e, e^{\prime} \in S^{n-1}, p, p^{\prime} \in \mathbb{R}^{n}$,

$$
\operatorname{diam}\left(T_{e}^{\delta}(p) \cap T_{e^{\prime}}^{\delta}\left(p^{\prime}\right)\right) \lesssim \frac{\delta}{\left|e-e^{\prime}\right|} .
$$

The idea is now the following: The two planes $H_{p}$ and $H_{q}, p \neq q$, intersect either in a line or not at all (if they are parallel). As for the neighbourhoods, if $|(x, y)|$ is large, the angle between $N_{p}$ and $N_{q}$ is large, and the intersection of the neighbourhoods of the planes is a small neighbourhood of the line along which the planes intersect. If $|(x, y)|$ is small and $|t|$ is large, the intersection line lies outside $B(0,2 R)$ and for $\delta$ small enough, also the neighbourhoods do not intersect inside $B(0,2 R)$. Another case, namely that $|(x, y)|$ and $|t|$ are small at the same time, cannot occur since $\|q\|_{H}$ is bounded away from zero.

Let us now make the various cases more precise. By $\sigma\left(N_{p}, N_{q}\right)$ we denote the angle between $N_{p}$ and $N_{q}$, so that, after choosing orientation, $\sigma\left(N_{p}, N_{q}\right) \in\left(0, \frac{\pi}{2}\right)$.

First assume $|(x, y)|>\delta^{\kappa+\frac{1}{8}}$. Then

$$
N_{p} \cdot N_{q}=\frac{1}{\sqrt{4|(x, y)|^{2}+1}}<\frac{1}{\sqrt{4 \delta^{2 \kappa+\frac{1}{4}}+1}} .
$$

We know that $H_{p}$ and $H_{q}$ intersect in a line. Consider a point on that line and the plane containing this point which is perpendicular to the intersection line. This plane $P$ is spanned by $N_{p}$ and $N_{q}$. Restricted to $P \cap B(0,2 R)$, the intersection of neighbourhoods of $H_{p}$ and $H_{q}$ corresponds to the intersection of rectangles (tubes) in a set of diameter (with respect to Euclidean metric) $\lesssim \delta^{1 / 4} / \delta^{\kappa+\frac{1}{8}}$. The whole intersection is contained in a $\lesssim \delta^{1 / 4} / \delta^{\kappa+\frac{1}{8}}$-tube. By (1.1) this is then contained in $\mathrm{a} \lesssim c_{2} \delta^{\frac{1}{16}-\frac{\kappa}{2}}$-neighbourhood with respect to $d_{H}$.

Assume now that $|(x, y)|<\delta^{\kappa+\frac{1}{8}}$ and $|t|>\delta^{2 \kappa}$. In this case, the intersection $N_{E}\left(H_{p},\left(c^{\prime} / c_{1}\right) \delta^{\frac{1}{4}}\right) \cap N_{E}\left(H_{q},\left(c^{\prime} / c_{1}\right) \delta^{\frac{1}{4}}\right)$ might actually be quite large, as $N_{p}$ and $N_{q}$ lie very close in orientation. However, $|t|$ is large enough to ensure that for small 
enough $\delta>0$, this intersection happens only outside $B(0,2 R)$. Suppose towards a contradiction that there exists a point $p^{\prime}=\left(x^{\prime}, y^{\prime}, t^{\prime}\right) \in N_{E}\left(H_{p},\left(c^{\prime} / c_{1}\right) \delta^{\frac{1}{4}}\right) \cap$ $N_{E}\left(H_{q},\left(c^{\prime} / c_{1}\right) \delta^{\frac{1}{4}}\right) \cap B(0,2 R)$. Since $p^{\prime}$ is contained in the neighbourhood of the $(x, y)$-plane, we find

$$
\left|t^{\prime}\right| \leq \frac{c^{\prime}}{c_{1}} \delta^{\frac{1}{4}}
$$

On the other hand, $p^{\prime} \in N_{E}\left(H_{q},\left(c^{\prime} / c_{1}\right) \delta^{\frac{1}{4}}\right)$ implies that $p^{\prime}$ lies on a plane parallel and close to $H_{q}$, that is, it has to fulfil the equation

$$
-2 y x^{\prime}+2 x y^{\prime}+t^{\prime}-t_{*}=0
$$

for some $t_{*}$ so that the corresponding plane is contained in $N_{E}\left(H_{q},\left(c^{\prime} / c_{1}\right) \delta^{1 / 4}\right)$. Simple trigonometry using the fact that

$$
N_{p} \cdot N_{q} \geq \frac{1}{\sqrt{4 \delta^{2 \kappa+\frac{1}{4}}+1}} \geq \frac{1}{\sqrt{5}}
$$

for $\delta \leq 1$, gives $\left|t-t_{*}\right| \leq \sqrt{5} \frac{c^{\prime}}{c_{1}} \delta^{\frac{1}{4}}$. We deduce

$$
\left|t^{\prime}\right| \geq\left|t_{*}\right|-2\left|\left(x^{\prime}, y^{\prime}\right) \cdot(y,-x)\right|>\delta^{2 \kappa}-\sqrt{5} \frac{c^{\prime}}{c_{1}} \delta^{\frac{1}{4}}-4 R \delta^{\kappa+\frac{1}{8}} .
$$

By assumption, $\kappa<1 / 8$, and this shows that (3.13) and (3.14) are incompatible for small enough $\delta$. Hence, in this case,

$$
N_{E}\left(H_{p},\left(c^{\prime} / c_{1}\right) \delta^{\frac{1}{4}}\right) \cap N_{E}\left(H_{q},\left(c^{\prime} / c_{1}\right) \delta^{\frac{1}{4}}\right) \cap B(0,2 R)=\emptyset .
$$

The last possibility would be that $|(x, y)|<\delta^{\kappa+\frac{1}{8}}$ and $|t|<\delta^{2 \kappa}$, but this cannot occur since then we would have

$$
\|q\|_{H}<\sqrt[4]{\delta^{4 \kappa+\frac{1}{2}}+\delta^{4 \kappa}}=\delta^{\kappa} \sqrt[4]{\delta^{\frac{1}{2}}+1}<2 \delta^{\kappa} .
$$

This concludes the proof of Proposition 3.8.

We now provide two lemmata which together will guarantee that the neighbourhood of a line has small $\mu$ mass.

Lemma 3.10. There exists a finite constant $C=C\left(\mathbb{H}^{1}\right)>0$ such that for any $\varepsilon>0$ and for any set $\ell \subset \mathbb{H}^{1}$ for which there exist points $p_{1}, \ldots, p_{N} \in \ell$ such that

$$
\ell \subseteq \bigcup_{i=1}^{N} B\left(p_{i}, \varepsilon\right)
$$

the neighbourhood $N_{H}(\ell, \varepsilon)$ can be covered by $\leq C \cdot N$ Korányi balls of radius $\varepsilon$. 
Proof. Observe that

$$
N_{H}(\ell, \varepsilon) \subset \bigcup_{i=1}^{N} B\left(p_{i}, 2 \varepsilon\right)
$$

and exploit the fact that $\left(\mathbb{H}^{1}, d_{H}\right)$ is doubling with constant $C$, so that we need at most $C$ balls of radius $\varepsilon$ in order to cover one of the balls $B\left(p_{i}, 2 \varepsilon\right)$.

Lemma 3.11. Let $\ell$ be a non-vertical line in $\mathbb{H}^{1}$. Then $\ell \cap B(0, R)$ can be covered by $\lesssim \varepsilon^{-2}$ balls of radius $\varepsilon$, where the implicit constant appearing in the number of balls is allowed to depend on $R$.

Proof. The line segment $\ell \cap B(0, R)$ can be partitioned in $\lesssim \varepsilon^{-2}$ intervals of length $\frac{\varepsilon^{2}}{4 c_{2}^{2}}$, where $c_{2}$ is chosen as in (1.1). Each such interval is contained in a Heisenberg ball of radius $\varepsilon$ centred at the mid-point.

Proposition 3.8, Lemma 3.10 and Lemma 3.11 yield together the following estimate.

Corollary 3.12. Let $p$ and $q$ be as in Proposition 3.8. Then

$$
\mu\left(\left(p * \mathcal{C}^{\prime}\right) \cap\left(q * \mathcal{C}^{\prime}\right)\right) \lesssim \delta^{\gamma(s-2)} .
$$

Recall that we have found earlier points $p_{1}$ and $p_{2}$ such that (3.10) holds. However, by our choice of $\kappa<\frac{s-2}{8 s}$, we obtain $\kappa<\gamma(s-2)$ with $\gamma=\frac{1}{16}-\frac{\kappa}{2}$, and so (3.10) is incompatible with (3.15). This contradiction shows that the original assumption $\operatorname{dim}_{H} B_{\theta}<\sigma$ for $\theta$ in some set of positive length cannot be valid, which concludes the proof of Theorem 0.3 .

\section{Concluding remarks and open questions}

Question 4.1. What is the sharp lower bound for randomized vertical projections akin to Theorem 0.2 for sets of dimension more than two?

Similarly as in [1,2], for integrability reasons it is not possible to derive better lower bounds for sets of larger dimension and it seems that a different approach would be needed in order to answer the above question. Such a different approach has been applied in this paper to the usual family of vertical Heisenberg projections and it has left us with the following question.

Question 4.2. Is it possible to give an improved lower bound for vertical projections in the spirit of Theorem 0.3 also for sets $B$ of dimension less than two?

The argument in Section 3 could be carried out also in the case $1<s<2$, except for the very last step: arbitrarily many left-translated copies of the $(x, y)$-plane may intersect along a non-horizontal (in the sense of sub-Riemannian geometry) line. Such a line has Hausdorff dimension 2, and it could therefore well support a 
Frostman measure $\mu$ of exponent $s<2$. In fact, if we translate by points $p_{1}, p_{2}, \ldots$ which lie in a small tube around a line, the intersection of the corresponding neighborhoods of planes will contain an open neighborhood of a line and hence there is no mass bound for this intersection that could be used to arrive at a contradiction. We remark that a similar issue occurs when dealing with restricted families of projections onto lines in $\mathbb{R}^{3}$, but in [17] a trick is shown to avoid this problematic situation by considering only a subfamily of lines. Unfortunately, this approach cannot be adopted here since - no matter how small we choose an interval $E \subset[0, \pi)-$ arbitrarily many distinct left translates of $\bigcup_{\theta \in E} \mathbb{V}_{\theta}$ may intersect a fixed line in a set of positive length.

If not for Hausdorff dimension, a lower bound of the form $1+\alpha(s)$ for sets with $\operatorname{dim}_{\mathrm{H}} B=s \in[1,2]$ might be proved for other notions of dimension.

Question 4.3. Is it possible to give an improved lower bound for the packing or box-counting dimension of images of a set $B$ with dimension less than two?

An analogous problem for special restricted families of orthogonal projections in $\mathbb{R}^{3}$ has been studied in [7]. If one wanted to apply the same strategy here, roughly speaking one would need that $d_{H}(p, q) \lesssim d_{H}\left(P_{\mathbb{V}_{\theta}^{\perp}}(p), P_{\mathbb{V}_{\theta}^{\perp}}(q)\right)$ whenever the line connecting $p$ and $q$ is 'angularly separated' from $V_{\theta}$, but this does not have to be the case.

\section{References}

[1] Z. Balogh, E. Durand-Cartagena, K. Fässler, P. Mattila and J. Tyson, The effect of projections on dimension in the Heisenberg group, Rev. Mat. Iberoam. 29 (2013), 381-432.

[2] Z. Balogh, K. Fässler, P. Mattila and J. Tyson, Projection and slicing theorems in Heisenberg groups, Adv. Math. 231 (2012), 569-604.

[3] Z. Balogh, M. Rickly and F. Serra CASSAno, Comparison of Hausdorff measures with respect to the Euclidean and the Heisenberg metric, Publ. Mat. 47 (2003), 237-259.

[4] Z. M. BALOGH, J. T. TYSON and K. WILDRICK, Frequency of Sobolev dimension distortion of horizontal subgroups of Heisenberg groups, preprint, arXiv:1303.7094

[5] J. Bourgain, The discretised sum-product and projection theorems, J. Analyse Math 112 (2010), 193-236.

[6] M. CHRIST, Hilbert transforms along curves. I. Nilpotent groups, Ann. of Math. (2) 122 (1985), 575-596.

[7] K. FÄSSLER and T. ORPONEN, On restricted families of projections in $\mathbb{R}^{3}$, Proc. Lond. Math. Soc. (3) 109 (2014), 353-381.

[8] I. HAHLOMAA, A sufficient condition for having big pieces of biLipschitz images of subsets of Euclidean space in Heisenberg groups, preprint, arXiv: 1212.0687

[9] R. Hovila, Transversality of isotropic projections, unrectifiability and Heisenberg groups, Rev. Mat. Iberoam. 30 (2014), 463-476.

[10] R. KaUfman, On Hausdorff dimension of projections, Mathematika 15 (1968), 153155.

[11] V. Magnani, Towards differential calculus in stratified groups J. Aust. Math. Soc. (1) 95 (2013), 76-128.

[12] J.M. MARSTRAND, Some fundamental geometrical properties of plane sets of fractional dimensions, Proc. Lond. Math. Soc. (3) 4 (1954), 257-302. 
[13] P. MattiLA, Hausdorff dimension, orthogonal projections and intersections with planes, Ann. Acad. Sci. Fenn. Ser A I Math 1 (1975), 227-244.

[14] P. Mattila, "Geometry of Sets and Measures in Euclidean Spaces", Cambridge University Press, 1995.

[15] P. Mattila, R. P. Serapioni and F. Serra Cassano, Characterizations of intrinsic rectifiability in Heisenberg groups, Ann. Sc. Norm. Super. Pisa Cl. Sci. (5) 4 (2010), 687-723.

[16] D. Oberlin and R. OBERLIn, Application of a Fourier restriction theorem to certain families of projections in $\mathbb{R}^{3}$, J. Geom. Anal. 25 (2015), 1476-1491.

[17] T. ORPONEN, Hausdorff dimension estimates for some restricted families of projections in $\mathbb{R}^{3}$, Adv. Math. 275 (2015), 147-183.

Mathematical Institute

University of Bern

Sidlerstrasse 5

CH-3012 Bern, Switzerland

katrin.faessler@math.unibe.ch

Department of Mathematics and Statistics University of Helsinki

P.O.B. 68

FI-00014 Helsinki, Finland

risto.hovila@helsinki.fi 